\title{
Motion adaptation and attention: A critical review and meta-analysis
}

\section{Article}

\section{Accepted Version}

Creative Commons: Attribution-Noncommercial-No Derivative Works 4.0

Bartlett, L. K., Graf, E. W., Hedger, N. and Adams, W. J. (2019) Motion adaptation and attention: A critical review and meta-analysis. Neuroscience and Biobehavioral Reviews, 96. pp. 290-301. ISSN 0149-7634 doi:

https://doi.org/10.1016/j.neubiorev.2018.10.010 Available at https://centaur.reading.ac.uk/87282/

It is advisable to refer to the publisher's version if you intend to cite from the work. See Guidance on citing.

To link to this article DOI: http://dx.doi.org/10.1016/j.neubiorev.2018.10.010

Publisher: Elsevier

All outputs in CentAUR are protected by Intellectual Property Rights law, including copyright law. Copyright and IPR is retained by the creators or other copyright holders. Terms and conditions for use of this material are defined in the End User Agreement.

\section{www.reading.ac.uk/centaur}

\section{CentAUR}

Central Archive at the University of Reading 
Reading's research outputs online 
See discussions, stats, and author profiles for this publication at: https://www.researchgate.net/publication/328383418

\section{Motion adaptation and attention: A critical review and meta-analysis}

Article in Neuroscience \& Biobehavioral Reviews · October 2018

DOl: 10.1016/j.neubiorev.2018.10.010

CITATIONS

0

4 authors:

Laura Bartlett

University of Southampton

4 PUBLICATIONS 2 CITATIONS

SEE PROFILE

Nicholas Hedger

University of Reading

19 PUBLICATIONS 232 CITATIONS

SEE PROFILE
READS

128

Erich Graf

University of Southampton

81 PUBLICATIONS 903 CITATIONS

SEE PROFILE

Wendy Jo Adams

University of Southampton

99 PUBLICATIONS 1,198 CITATIONS

SEE PROFILE

Some of the authors of this publication are also working on these related projects:

Perceptual Aftereffects and Attention View project

Project Visual Illusions and Awareness View project 


\title{
Motion adaptation and attention: A critical review and meta- analysis.
}

\author{
Laura Bartlett ${ }^{a}$, Erich W. Graf ${ }^{a}$, Nicholas Hedger ${ }^{\text {ab }}$, Wendy J. Adams ${ }^{a}$ \\ Psychology, University of Southampton, UK
}

"Psychology, FSHS, University of Southampton, Southampton, SO17 1BJ, England

${ }^{\circ}$ Present address: School of Psychology \& Clinical Language Sciences, University of

Reading, RG6 6AL, England

Running head: Motion adaptation and attention: A critical review and meta-analysis.

* Corresponding author at: Psychology, FSHS, University of Southampton, Southampton, SO17 1BJ, England

E-mail address: w.adams@soton.ac.uk (W.J. Adams).

This research did not receive any specific grant from funding agencies in the public, commercial, or not-for-profit sectors. 
1 BARTLETT, L., E. W. Graf, N. Hedger, and W. J. Adams. Motion adaptation and attention:

2 A critical review and meta-analysis. NEUROSCI BIOBEHAV REV XXX-XXX, 2018.-

4 The motion aftereffect (MAE) provides a behavioural probe into the mechanisms underlying

5 motion perception, and has been used to study the effects of attention on motion processing.

6 Visual attention can enhance detection and discrimination of selected visual signals.

7 However, the relationship between attention and motion processing remains contentious: not

8 all studies find that attention increases MAEs. Our meta-analysis reveals several factors that

9 explain superficially discrepant findings.

10

11 Across studies (37 independent samples, 76 effects) motion adaptation was significantly and

12 substantially enhanced by attention (Cohen's $d=1.12, p<.0001$ ). The effect more than doubled

13 when adapting to translating (vs. expanding or rotating) motion. Other factors affecting the

14 attention-MAE relationship included stimulus size, eccentricity and speed. By considering

15 these behavioural analyses alongside neurophysiological work, we conclude that feature-

16 based (rather than spatial, or object-based) attention is the biggest driver of sensory

17 adaptation.

18

19 Comparisons between naïve and non-naïve observers, different response paradigms, and

20 assessment of 'file-drawer effects' indicate that neither response bias nor publication bias are

21 likely to have significantly inflated the estimated effect of attention.

22

23 Keywords:

24 Attention, motion adaptation, motion aftereffect, systematic review, meta-analysis 


\section{Background}

Attention refers to our ability to selectively process certain aspects of a visual scene, such

that particular regions or features are enhanced, and irrelevant stimuli are inhibited (Carrasco,

2011). The effects of attention on perceptual processes are usually measured via

manipulations of covert attention in which attention is directed independently of eye

movements. This contrasts with overt attention, in which a redirection of attention is

enhances signals from the attended location, reducing noise, and changing decision criteria

34 (see Carrasco, 2011 for an overview), and has been shown to operate at all levels of the visual

35 hierarchy, even early visual areas previously believed to be pre-attentive and entirely sensory, i.e. the primary visual cortex (V1) (e.g. Silver et al., 2007; Somers et al., 1999). Whether such attentional effects generalize to motion processing, however, remains contentious. Here we consider evidence for the influence of covert attention on motion processing, as evidenced by effects of attention on motion adaptation.

40 The relationship between attention and visual motion processing has been studied since the early $20^{\text {th }}$ century. Wertheimer $(1912 / 1961)$ found that attention altered the perceived motion direction of ambiguous apparent motion, a finding supported by more recent work

43 (Kohler, Haddad, Singer, \& Muckli, 2008). In addition, researchers have found that the 44 perceived direction of third-order motion (the motion of important visual details, i.e. the 45 'figure' is separated from the 'ground' within a salience map; Lu and Sperling, 2001) can be determined entirely by attention (Lu and Sperling, 1995). The observed effect of attention on

47 higher-level motion processes is in keeping with the notion that attention exerts a greater 48 influence in more advanced regions of the cortical visual hierarchy (Carrasco, 2011).

49 However, the picture is less clear when we consider low-level (first-order / luminance50 defined) motion. 

back to Aristotle, and it was popularised by Addams (1834) as the 'the waterfall effect'.

57 Fig. 1a).

58

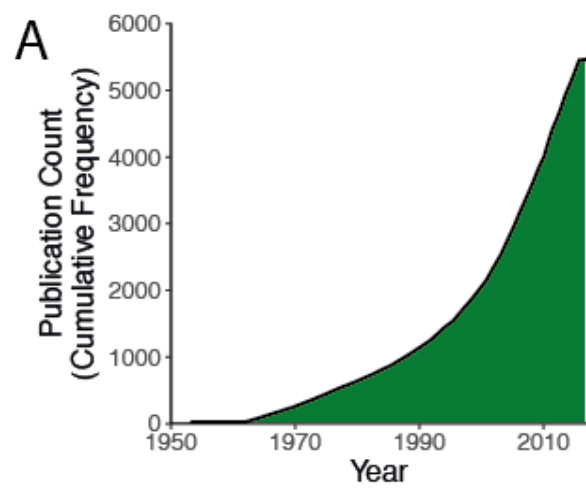

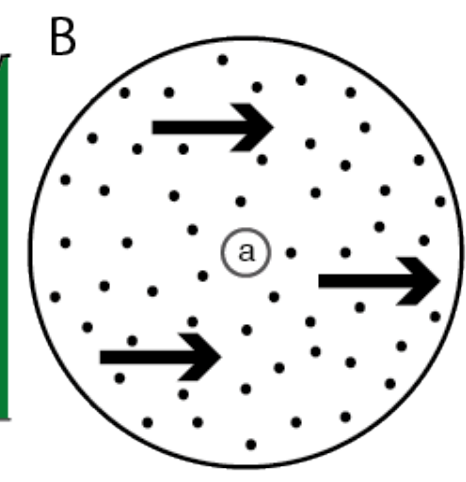

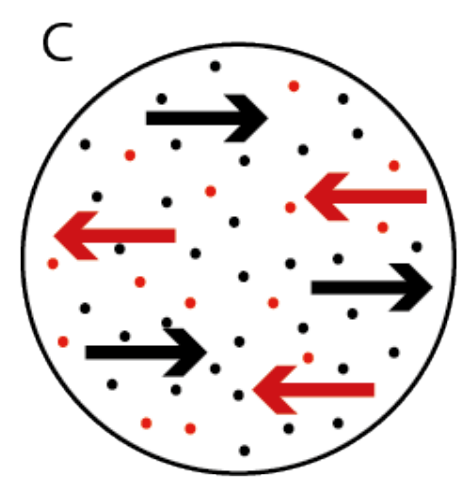

Fig. 1. (a) Cumulative frequency of publications related to the motion aftereffect. A PubMed search (keywords: motion aftereffect OR motion adaptation) revealed 5470 publications since 1950. (b \& c) A schematic of typical paradigms used to measure the effect of covert attention on motion adaptation. (b) A moving stimulus is presented within an annulus. To manipulate attention, a centrally presented stimulus such as a stream of letters is fixated, while observers perform either a difficult / high-load task, or an easy / low-load task, or passively view the stimulus. (c) The motion tracking paradigm: two populations of dots (indicated here by different colours) each follow a different motion trajectory. Observers attend to one of the two superimposed motion patterns.

If basic motion mechanisms are affected by spatial attention, we expect to find larger

70 MAEs when attention is directed towards, rather than away from a moving adaptation 
that diverting attention to a central rapid serial visual presentation (RSVP) task had no effect on adaptation to a spiral motion stimulus, leading to a long-held view that the mechanisms responsible for motion adaptation are pre-attentive. Indeed, some recent papers continue to endorse this view (Morgan, 2013, 2012, 2011). This suggestion - that motion adaptation is independent of attention - is aligned with previous reports that adaptation to other simple visual features (spatial frequency, orientation) is unaffected by attention or awareness (Blake \& Fox, 1974; He, Cavanagh, \& Intriligator, 1996).

A landmark study by Chaudhuri (1990) challenged this pervasive view. Observers viewed a large moving stimulus for 60 seconds. During this adaptation period, numbers and letters appeared within a small central aperture and observers either engaged in a demanding alphanumeric task, or passively viewed the stimulus (see Fig. 1b). Observers subsequently viewed a static stimulus and reported the MAE duration. Attending to the central task caused a substantial reduction in the duration of the subsequent motion after-effect. Subsequently, multiple studies have similarly found that diverting attention away from a moving adaptor reduces the duration (e.g. Chaudhuri, 1990; Rezec, Krekelberg, \& Dobkins, 2004) or velocity of the subsequent MAE (e.g. Georgiades \& Harris, 2000a; Taya, Adams, Graf, \& Lavie, 2009).

$$
\text { In broad agreement with this behavioural data, neuroimaging evidence suggests that, }
$$
under similar attentional manipulations, motion-related activity in medial temporal (MT) / medial superior temporal (MST) areas is modulated by attention (Beauchamp et al., 1997; Rees et al., 1997). Load theory of selective attention suggests that during a demanding task, limited resources are available to process task-irrelevant information (such as a peripheral motion stimulus). Conversely, a low-load task requiring fewer resources leads to greater processing of task irrelevant stimuli (Lavie, 2005). Rees, Frith, \& Lavie (1997) presented irrelevant expanding motion in the periphery and words at fixation. In line with attentional 
97 load theory, a low load task (detect uppercase words) resulted in increased activation across several areas (MT, V1/V2 and the superior colliculus) and longer MAE durations, relative to a high-load task (detect bisyllabic words).

A clear consensus on whether (or under what conditions) attention increases motion

101 adaptation is yet to emerge, however, due to null (Morgan, 2013, 2012, 2011; Pavan and

102 Greenlee, 2015), or inconsistent findings (e.g. Georgiades \& Harris, 2002b; Takeuchi \& Kita,

103 1994). One might argue that, given the importance of motion for fundamental tasks such as

104 segmentation (including breaking camouflage), depth perception and guiding self-motion

105 (e.g. Gibson, 1958; Nakayama, 1985), it would be evolutionarily advantageous if motion

106 were processed independently of attention. Certainly, motion is a powerful cue for pop-out in

107 visual search (Driver et al., 1992; Nakayama and Silverman, 1986). If motion adaptation

108 mechanisms are pre-attentive, why have many studies reported an effect of attention on

109 MAEs? One suggestion is that affirmative findings reflect response bias: observers expect

110 weaker MAEs to follow diverted-attention conditions and bias their responses accordingly.

111 Measures of MAE duration (as used by Chaudhuri, 1990 and others) may be particularly susceptible to bias / criterion effects because observers struggle to determine the point at

113 which the MAE has completely disappeared (Morgan, 2012; Blake \& Hiris, 1993).

114 Other factors, such as the choice of stimuli and experimental paradigm almost

115 certainly contribute to variability across the findings from different studies. Paradigms differ

116 in the extent to which the attentional manipulations direct spatial, featural and surface /

117 object-based attention. As reviewed below (see section 1.2.1.1), a coherently translating 118 stimulus may recruit feature-based attention more effectively than complex (rotating and /or 119 expanding) stimuli. Others have demonstrated that the eccentricity of the adaptor (Georgiades 120 and Harris, 2000a, 2000b) and the type of test stimulus (static vs. dynamic; Culham, 121 Verstraten, Ashida, \& Cavanagh, 2000) may modulate the effect of attention. Attentional 
tracking paradigms, in which observers attend one of two superimposed motion stimuli (e.g.

123 Lankheet \& Verstraten, 1995; Mukai \& Watanabe, 2001; see Fig. 1c) may reveal larger

124 attentional effects than those that direct attention towards or away from the location of a

125 single motion stimulus (Morgan, 2011). A meta-analysis allows us to evaluate all of these

126 factors and others (as detailed in Section 1.2) such that we can better understand whether, and 127 under what conditions, motion processing (and motion adaptation) is modulated by attention.

\subsection{The current review: justification and objectives}

130 In contrast to single empirical papers, or selective, narrative reviews (e.g. Burr \&

131 Thompson, 2011), our meta-analysis provides sufficient power to quantify the effects of 132 multiple factors on the attention-motion relationship. We assess the effects of varying the 133 adaptation stimulus (translation vs. complex motion, size, eccentricity, speed and duration),

134 the test stimulus (static vs. dynamic), the experimental paradigm (MAE duration vs.

135 strength/speed, 2AFC vs. matching, attentional tracking vs. distraction) and participant 136 characteristics (naïve vs. non-naïve).

138 1.2. Factors that may affect the relationship between attention and motion processing

139 1.2.1. Characteristics of the adaptation stimulus

140 1.2.1.1. Type of motion

The effect of attention on neural responses to motion stimuli may depend on the type

142 of motion being presented. Spatial attention has been shown to increase the overall response

143 gain of MT neurons corresponding to the attended region (Treue and Martínez-Trujillo,

144 1999). In contrast, feature-based attention has a substantial direction-specific effect, such that

145 neurons in V1, MT and MST tuned to the attended motion direction show a response gain,

146 while neurons tuned to the opposite direction are suppressed (Saproo and Serences, 2014; 
147 Treue and Martínez-Trujillo, 1999; Treue and Maunsell, 1996). Importantly, this effect of

148 feature-based attention spreads across the visual field, such that attending to leftward motion

149 in one location will enhance processing of leftward motion across all retinal locations. One

150 would expect this direction-specific modulation of neural activation to have a large role in the

151 enhancement of MAEs. When observers attend to a large, coherently translating stimulus

152 (vs. central letters, for example), spatial and feature-based attention will combine (Treue and

153 Martínez-Trujillo, 1999) to enhance the neural representation of the moving stimulus across

154 multiple motion-sensitive cortical regions.

155 In contrast to translating stimuli, rotating or expanding motion patterns are composed

156 of local motion signals whose directions vary as a function of position (Carrasco, 2011).

157 Attending to these complex motion stimuli will not, therefore, enhance V1/MT activity via

158 simple feature-based attention mechanisms. However, there is now evidence from

159 neurophysiology (Wannig et al., 2007) and fMRI (Kamitani and Tong, 2006) that surface, or

160 object-based attention can enhance V1 and MT activation as a function of both direction and

161 position, when more complex motion patterns are attended. These effects could be driven by

162 feedback from the dorsal section of MST (MSTd), an area known to have larger and more

163 complex receptive fields that have been implicated in optic flow processing (Graziano et al.,

164 1994; Saito et al., 1986; Tanaka and Saito, 1989).

165 Behavioural studies of attention and motion processing often assume, implicitly, that increased motion-related activation (due to attention) can be inferred from changes in

167 adaptation. However, adaptation effects may vary across regions. Following prolonged

168 activation, V1 neurons show substantial reductions in responsiveness and sensitivity to

169 motion directions close to the adapted direction. However, reductions in responsivity in MT

170 are much smaller (Kohn and Movshon, 2004, 2003). Thus, differences in adaptation across

171 the cortex, in addition to variations in the effects of spatial, featural and surface-based 
attention on neural responses to translating vs. complex motion patterns, strongly suggest that the effect of attention on adaptation will depend on the type of motion stimulus.

174

175

176

177

178

179

180

181

182

183

184

185

186

187

188

189

190

191

\subsubsection{Size of adaptation stimuli}

Previous studies have asked whether stimulus size determines the extent of attentional modulation (Georgiades and Harris, 2000b; Takeuchi and Kita, 1994). If attentional effects are larger at higher-level cortical regions (at least for complex motion) then attentional modulation of MAEs might increase with stimulus size, given increasing receptive field size from V1 to MT and MST (Smith et al., 2001). On the other hand, it may be easier to shift spatial attention away from a smaller stimulus, particularly as its distance from the taskrelevant stimulus increases.

\subsubsection{Eccentricity of adaptation stimuli}

Most paradigms used to explore the attention-motion relationship use an attentional task related to a central stimulus to draw attention away from a peripheral motion stimulus. One might expect that when the adaptation stimulus is close to the distracting stimulus, attentional resources may 'spill over' to the motion stimulus more easily. A key component of the load theory of attention is that the target and distractor must be spatially separated (Lavie, 2005). A demanding task at fixation may reduce the extent to which peripheral distractors are processed, by narrowing the window of spatial attention around the central, task relevant stimuli. Thus, more eccentric adaptation stimuli may reveal larger effects of attentional modulation. Conversely, there is some empirical evidence that attentional modulation is stronger for adaptation stimuli closer to fixation (Georgiades and Harris, 2000a, 2000b). 


\subsubsection{Speed of adaptation stimuli}

Many studies have considered how motion adaptation varies as a function of adaptor speed, classically reported to follow an inverted U-shape (see Thompson, 1998 for an overview). In terms of attention, Georgiades \& Harris (2002b) found that attention modulated motion adaptation for faster adaptation stimuli. However, for slower adaptation stimuli, the effect of attention increased with spatial frequency. The relationship between speed, attention, and motion processing has rarely been examined within studies. However, the wide range of adaptation speeds used across different studies allows us to explore this in the current analysis.

\subsubsection{Adaptation duration}

As adaptation duration increases, MAEs increase (Hershenson, 1993). In previous work, we explored how the effects of attention vary across the adaptation period by measuring the MAE at regular intervals during an extended adaptation period (Bartlett et al., 2018). Attention affected the asymptotic MAE magnitude, but not the rate at which it accumulated (i.e. the time constant). This finding is broadly consistent with Takeuchi \& Kita (1994), who found that diverted attention led to reduced MAEs across adaptation durations of 20, 40 and 80 seconds.

It could be argued, however, that diverting attention may reduce the rate of adaptation, without affecting the asymptotic point, i.e. at longer durations adaptation is saturated, and will not be further enhanced by attention. Such an effect could account for some null findings in the literature, and aligns with work by Blake and colleagues regarding perceptual awareness: after-effects in motion or spatial frequency are reduced under suppression or crowding for low contrast adaptors only - adaptation reaches saturation at higher contrasts, irrespective of awareness (Blake et al., 2006; Blake and Fox, 1974). 


\subsubsection{Static vs. dynamic test stimuli}

Motion adaptation can be quantified using static test stimuli (e.g. Chaudhuri, 1990) or

226 dynamic tests, such as random dot motion patterns (e.g. Mukai and Watanabe, 2001; Taya et al., 2009) or counterphase flicker (e.g. Nishida and Ashida, 2000; Rezec et al., 2004). Nishida

\& Sato (1995) suggested that static test stimuli reflect adaptation to first order motion, whereas dynamic (flicker) tests reveal second order MAEs. Static and dynamic tests may

230 also differ in terms of sensitivity to monocular vs. binocular motion mechanisms (e.g.

231 Nishida \& Ashida, 2000), and to storage effects (e.g. Verstraten, Fredericksen, Van Wezel, 232 Lankheet, \& Van De Grind, 1996). Using an attentional tracking paradigm (see Fig. 1c),

233 Culham, Verstraten, Ashida, \& Cavanagh (2000) reported that attention modulated the MAE

234 only when measured via a dynamic test. In summary, MAEs obtained from static and

235 dynamic test stimuli may reflect different motion mechanisms that are differentially 236 modulated by attention.

\subsubsection{Experimental paradigm}

\subsubsection{MAE measurement and response}

Many studies have quantified the effects of attention on motion adaptation by asking observers to report the cessation of the (illusory) motion of a static test stimulus, i.e. the MAE

242 duration (Chaudhuri, 1990; Morgan, 2012; Rezec et al., 2004). As noted above, this reporting

243 method has been criticised as susceptible to response bias. Alternatively, MAE magnitude

244 has been quantified via velocity matching (Georgiades and Harris, 2000a). 2AFC designs

245 have also been implemented, in which participants select one of two response options, such

246 as the test stimulus' motion direction (e.g. Kaunitz, Fracasso, \& Melcher, 2011; Taya et al., 
247 2009) or which of two test stimuli was moving faster (Morgan, 2013). Some 2AFC

248 paradigms use nulling techniques in which the percentage of test stimulus dots moving

249 oppositely to the aftereffect (Blake \& Hiris, 1993; e.g. Mukai \& Watanabe, 2001) or the

250 phase shift of the test (e.g. Culham et al., 2000) is adjusted to counteract the aftereffect.

251 2AFC / two interval forced choice (2IFC) designs have been considered preferable to

252 duration or matching tasks in terms of minimising response bias (Morgan, 2013).

253 If our analyses reveal that the reported effects of attention on motion adaptation are

254 larger when measured via duration estimation or velocity matching paradigms, this would

255 suggest that some reported effects have been inflated by response bias.

\subsubsection{Attentional manipulation}

Two distinct methods of manipulating attention have been used in the context of

motion adaptation. 'Distractor' paradigms involve diverting attention away from a moving

260 adaptation stimulus - usually towards a centrally presented, difficult task (see Fig. 1b). This

261 condition is compared with one in which more attention is deployed to the adaptation

262 stimulus, for example during passive viewing. This paradigm involves spatial attention (the

263 moving adaptor and central task are spatially separated). Depending on the type of motion, it

264 may also involve feature and / or surface based attention, as discussed above. Some would

265 also consider it to involve 'dimension' based attention, i.e., attention to motion rather than

266 another visual dimension such as colour or shape, because the central task does not usually

267 rely on motion. Reductions in motion-related activation in V1 and MT/MST have been found

268 when attention is directed to the colour or luminance of a moving stimulus, rather than its

269 motion (Beauchamp et al., 1997; Saproo and Serences, 2014), with corresponding changes in

270 the resultant MAE also reported (Taya et al., 2009). 

superimposed motion patterns (see Fig. 1c). This relies on feature-based attention: i.e.

273 attention is directed to one motion direction, at the expense of another motion direction.

274 Studies that have used attentional tracking have fairly consistently demonstrated attentional 275 modulation of motion adaptation (e.g. Alais and Blake, 1999; Culham et al., 2000; Lankheet 276 and Verstraten, 1995). Accordingly, it has been suggested that the two paradigms probe 277 distinct attentional mechanisms that vary in their effects on motion processing (Morgan, $2782012,2011)$.

279

\subsubsection{Participant characteristics}

\subsubsection{Participant naivety}

It is often noted that the risk of response bias increases with certain paradigms (as

283 discussed in Section 1.2.3.1), and also with non-naïve observers who may know the research

284 hypotheses. As noted above, there have been claims that some reports of attentional 285 modulation of motion adaptation are not just inflated by, but due to response bias (Morgan, 286 2013, 2012). Indeed, Morgan (2012) failed to find attentional modulation of motion

287 adaptation with naïve observers. The overall picture, however, is less clear, with other studies 288 finding significant effects within groups of naïve observers (e.g. Chaudhuri, 1990; Patterson 289 et al., 2005).

2. Method

\section{2.1.Inclusion and coding decisions}

\section{2.1.1. Inclusion criteria}

294 All studies that met the following criteria were included in the present meta-analysis: 
1. The study manipulated attention during motion adaptation and reported the subsequent behavioural motion aftereffect.

2. The stimuli did not differ across attentional manipulations.

3. The study was published in an English language journal on or before August 2016.

4. Participants were healthy human adults - studies using patient populations were excluded.

5. The study was not a re-analysis of existing data

6. Sufficient information was provided in order to estimate an effect size (see section 2.3).

7. Only within subject designs were included, due to well-known issues equating within subject and between subject effect size measurements (Lakens, 2013). This removed

\subsubsection{Other coding and inclusion decisions}

1. If the study assessed both a low load and a no load (passive) condition, these data were pooled into a single 'low load' condition and compared against the high load condition.

2. If the study included a manipulation that was not pertinent to the research questions (e.g. different levels of contrast in the adapting stimulus; Rezec et al., 2004), data were pooled across this manipulation (see Supplementary Material S1 for a full list).

3. In order to reduce un-modelled variability, data from conditions / experiments with atypical presentation conditions were excluded. For example, in studies investigating interocular transfer, only data from conditions in which adaptation and test stimuli were presented to the same eye(s) were included (e.g. Nishida and Ashida, 2000). See 

excluded conditions.

4. If the study was investigating awareness, we only included the data from conditions where observers were aware of the adapter (e.g. Kaunitz et al., 2011).

5. Only visual manipulations of attention were included; auditory attention conditions were excluded (e.g. Houghton, Macken, \& Jones, 2003).

\subsection{General search and coding strategies}

One of the authors (LB) conducted the search for relevant studies and coded the data,

328 in consultation with all authors. A PubMed database search was first conducted.

329 Subsequently, the reference sections of all relevant studies were examined to identify

330 additional relevant papers. Next, articles citing any of the relevant studies were identified via

331 Google Scholar, and the reference lists of all of these articles were then examined. A

332 summary of the excluded articles and the database search terms are presented in the

333 Supplementary Material S2, according to the 'Preferred Reporting Items for Systematic 334 reviews and Meta Analysis' guidelines (PRISMA: Moher, Liberati, Tetzlaff, Altman, \& The 335 PRISMA Group, 2009).

\subsection{Methods}

2.3.1. Effect size metric

Cohen's $d$, the standardised difference between means (Cohen, 1977), was used as the

340 effect size index for all outcome measures. A positive value indicates a stronger motion

341 aftereffect following passive or low-load adaptation than diverted or high-load attention

342 during adaptation, or following motion-focused compared to passive viewing. 
346 difference between means ( $\left.\mathrm{M}_{\text {difi }}\right)$ standardised by the averaged standard deviation (SD) of the

347 measures (Lakens, 2013):

$348 \quad d_{a v}=\frac{M_{d i f f}}{\frac{S D_{1}+S D_{2}}{2}}$

Equation 1.

349 This formula is recommended for repeated measures designs where there is no pre-post distinction (e.g. before or after treatment). Because both SD measures are equally good estimators of population variability, averaging the two gives the best estimate (Cumming,

352 2012). In cases where standard deviations were only reported for multiple levels of an

353 irrelevant experimental variable, these values were pooled to reflect the standard deviation 354 collapsed across these levels (see Supplementary Material S3).

If means and standard deviations were not reported, effect sizes were computed from $t, p$ or $F$ values and the degrees of freedom to give $d_{R u}$, the difference between means standardised by the standard deviation of the difference scores (Lakens, 2013):

$d_{R M}=\frac{t}{\sqrt{N}} \times \sqrt{2(1-r)}$

Equation 2.

In practice, few effect sizes were calculated using $d_{R M}$ (13.16\% of overall). As

360 Equation 2 indicates, this estimate corrects for the paired correlation $(r)$ between conditions.

361 These correlations were calculated using equations reported by Morris \& DeShon (2002),

362 after first computing the variance of difference scores using reported $N, M_{\text {dir }}$ and $t$ values (see

363 Supplementary Material S4). Paired correlations were estimated for the five effects for which

364 the required data were available, however one was removed as it fell outside the possible

365 range, leaving 4 correlations in total $(\mathrm{M}=0.81, \mathrm{SD}=0.31)$. The mean of these correlations

366 was then assumed for the remaining calculations of $d_{R M}$. 
$368 S E=\sqrt{\frac{\frac{1}{N+d^{2}}}{2 N}}$

Equation 3.

369 As in Equation 2, this SE formula was multiplied by $\sqrt{2(1-r)}$ to correct for the paired 370 correlation between conditions.

371 When relevant statistics (e.g. $t$ or $F$ statistics) were not reported in the text, the effect

372 size was estimated, where possible, using means and standard deviations estimated from

373 published figures, via 'GraphClick' software (Arizona Software Inc., 2010). Finally, if

374 insufficient information was available from any source, the study was excluded from 375 analyses.

376

\subsection{Model and analysis decisions}

Effect size data were analysed in a random effects model. This model assumes that studies are estimating independent, randomly sampled values of the population parameters, and it is tolerant to heterogeneity across effect sizes (Cumming, 2012). Total effect size heterogeneity was estimated using the standardised measure Cochran's $Q$, while $I^{2}$ was used to estimate additional heterogeneity beyond that expected in a fixed effects model (Cumming, 2012). Parameter estimates were derived via restricted maximum likelihood estimation to minimise bias (Viechtbauer, 2005). To statistically assess model coefficients, Wald-type chi squared tests were computed.

386 The pseudo- $\mathrm{R}^{2}$ statistic was used to quantify the heterogeneity across effect sizes that was 387 explained by moderators (see Supplementary Material S5). The unstandardized regression coefficient $(b)$ indicated the direction and magnitude of effects. Likelihood ratio tests were used to compare models (whose parameters were derived via maximum likelihood estimation), in order to identify moderators that made a significant contribution to explained 
heterogeneity. All analyses were carried out in $R$, using the 'metafor' package (Viechtbauer, 2010).

\subsection{Dependency among effect sizes}

The number of included conditions (nested within samples) and the number of independent samples (nested within studies) were coded. In some cases, samples were exposed to many conditions, resulting in multiple effect sizes from a single group of participants. Collapsing the data across these effects would ignore important information. However, the contribution of multiple effect sizes by a sample introduces dependency in the data; the results of the meta-analysis can become biased towards the (correlated) effect size estimates due to a single unrepresentative sample. The influence of dependency was examined by creating multi-level models (Cheung, 2014) where conditions (level 2) were nested within their samples (level 3). This allowed us to determine whether there was a

404 significant effect size dependency (i.e. whether a 3-level model provides a better fit than a 2405 level model). In addition, we investigated the influence of dependency by creating resampled data sets that included one effect size from each independent sample. This allows an examination of the data under conditions where dependency is eliminated (Greenhouse and 408 Iyengar, 1994).

\section{Results}

\subsection{Summary of included data}

In total, 29 studies were analysed, involving 229 participants across 37 independent

413 samples, yielding 76 effect size estimates. Detailed information about each effect is available

414 in the Supplementary Material S1. Two effect sizes were more than \pm 3 SDs from the mean.

415 However, removing these outliers reduced the overall effect size by only 0.04 and all 
416 significant moderators remained significant. They were therefore included in the main

417 analysis. The coding for each moderator variable can be found in Table 1, and a summary of 418 moderator coding for each effect can be found in Supplementary Material S6.

419 
Table 1

\section{Moderator coding}

\begin{tabular}{|c|c|c|c|c|c|}
\hline Moderator & Type & Values & Description of variable & $\begin{array}{l}\text { Descriptive } \\
\text { Statistics* }\end{array}$ & $\begin{array}{l}\text { Missing } \\
\text { Cases }\end{array}$ \\
\hline \multicolumn{6}{|c|}{ Characteristics of adaptation stimulus } \\
\hline $\begin{array}{l}\text { Type of motion } \\
\text { (Section 1.2.1.1) }\end{array}$ & Categorical & $\begin{array}{l}1=\text { Translational } \\
\text { motion } \\
2=\text { Complex } \\
\text { motion }\end{array}$ & $\begin{array}{l}\text { The type of motion used for adaptation in the } \\
\text { study. Complex motion refers to any case where } \\
\text { motion direction varies across the stimulus (i.e. } \\
\text { expansion, contraction, rotation and spiral motion. }\end{array}$ & $\begin{array}{l}\mathrm{k}=76 \\
\mathrm{~N}_{1}=46 \\
\mathrm{~N}_{2}=30\end{array}$ & 0 \\
\hline $\begin{array}{l}\text { Adaptation stimulus area } \\
\text { (Section 1.2.1.2) }\end{array}$ & Continuous & $1.89^{\mathrm{o}_{2}-1256.39^{\mathrm{o}_{2}}}$ & $\begin{array}{l}\text { The total area (in degrees of visual angle }{ }^{2} \text { ) } \\
\text { covered by the adaptation stimulus. Excluded } \\
\text { those not reporting the size of a central blank } \\
\text { square/ellipse, or if adapting stimulus shape was } \\
\text { not clearly specified. }\end{array}$ & $\begin{array}{l}\mathrm{k}=64 \\
\mu=158.87^{\circ} \\
\sigma=277.50^{\circ_{2}} \\
\text { Range }=1.89^{\circ_{2}} \\
1256.39^{\circ}\end{array}$ & 12 \\
\hline $\begin{array}{l}\text { Eccentricity of adaptation } \\
\text { stimuli } \\
\text { (Section 1.2.1.3) }\end{array}$ & Continuous & $0.07^{\circ}-3.5^{\circ}$ & $\begin{array}{l}\text { The distance between fixation and the adaptation } \\
\text { stimulus (in degrees of visual angle). Averaged } \\
\text { across width and height of this space if they } \\
\text { differed. }\end{array}$ & $\begin{array}{l}\mathrm{k}=55 \\
\mu=1.03^{\circ} \\
\sigma=1.08^{\circ} \\
\text { Range }=0.07^{\circ}-5^{\circ}\end{array}$ & 21 \\
\hline $\begin{array}{l}\text { Speed of adaptation } \\
\text { stimuli } \\
\text { (Section 1.2.1.4) }\end{array}$ & Continuous & $0.6^{\circ} / \mathrm{sec}-8^{\circ} / \mathrm{sec}$ & $\begin{array}{l}\text { The speed of the adaptation stimulus. Calculated } \\
\text { tangential speed at average eccentricity for } \\
\text { rotational motion. }\end{array}$ & $\begin{array}{l}\mathrm{k}=45 \\
\mu=4.16^{\circ} / \mathrm{sec} \\
\sigma=1.79^{\circ} / \mathrm{sec}\end{array}$ & 31 \\
\hline $\begin{array}{l}\text { Duration of adaptation } \\
\text { (Section 1.2.1.5) }\end{array}$ & Continuous & $1 \mathrm{sec}-90 \mathrm{sec}$ & $\begin{array}{l}\text { The duration of a single adaptation period within } \\
\text { each trial. }\end{array}$ & $\begin{array}{l}\text { Range }=0.6^{\circ} / \mathrm{sec}- \\
8^{\circ} / \mathrm{sec} \\
\mathrm{k}=66 \\
\mu=40.11 \mathrm{sec} \\
\sigma=21.40 \mathrm{sec} \\
\text { Range }=1-90 \mathrm{sec}\end{array}$ & 10 \\
\hline \multicolumn{6}{|c|}{ Test stimulus characteristics } \\
\hline $\begin{array}{l}\text { Static vs. dynamic test } \\
\text { stimuli }\end{array}$ & Categorical & $\begin{array}{l}1=\text { Static } \\
2=\text { Dynamic }\end{array}$ & $\begin{array}{l}\text { The type of test stimulus used to measure the } \\
\text { MAE. }\end{array}$ & $\begin{array}{l}\mathrm{k}=76 \\
\mathrm{~N}_{1}=44\end{array}$ & 0 \\
\hline
\end{tabular}


(Section 1.2.2.1)

Experimental paradigm

MAE measurement

(Section 1.2.3.1)

MAE response

(Section 1.2.3.1)

Attentional manipulation

(Section 1.2.3.2)

Participant characteristics

Participant naivety

(Section 1.2.4.1)
Categorical $1=$ Duration $2=$ Strength

\section{Categorical 1=Magnitude} $2=2 \mathrm{AFC}$

Categorical $1=$ Distractor $2=$ Tracking

Categorical $1=$ Naïve $2=$ Other
The method of measuring the MAE. 'Duration' $\quad k=92$ paradigms record the time until MAE cessation. $\quad \mathrm{N}_{1}=51$ The 'strength' category includes all other methods $\mathrm{N}_{2}=25$ of MAE measurement (e.g. nulling, 2AFC).

2AFC measures of the MAE require participants $\quad \mathrm{k}=76$ to select one response of two response options $\quad \mathrm{N}_{1}=64$ (e.g. test is moving left or rightward). All other $\quad \mathrm{N}_{2}=12$ methods are coded as 'magnitude' responses.

The paradigm for manipulating attention.

Distractor paradigms (see Fig. 1b) divert attention $\quad \mathrm{N}_{1}=70$ from adapting motion with a centrally presented $\quad \mathrm{N}_{2}=6$ task. Attentional tracking (see Fig. 1c) involves attending to one of two superimposed motion directions.

'Naïve' refers to a subject group composed only $\quad \mathrm{k}=69$ of observers naïve to the research hypotheses. The $\mathrm{N}_{1}=44$ 'other' category includes experienced-only $\quad \mathrm{N}_{2}=25$ samples, as well as mixed naïve and experienced subject groups.

$* k$ refers to the number of effects; $N$ indicates the number of effects for each condition 
425 found $\left(k=76, N=229, d_{R M}=1.12,95 \%\right.$ CI $\left.[0.87,1.38], p<.001\right)$. A number of analyses were

426 conducted to explore potential file-drawer effects (in which null effects are sometimes

427 unpublished). Rosenthal's (1991) fail-safe $\mathrm{N}$ indicated that 7,767 additional, null studies

428 would be required to reduce the pooled effect size to non-significance. The actual number of

429 unpublished (negative effect) studies was estimated to be 7, using the trim and fill method,

430 based on the symmetry of the data (Duval and Tweedie, 2000a, 2000b). Attentional

431 modulation of the MAE remained significant when these (simulated) effects were included,

432 reducing the effect by only 0.18 . When any single contributing effect was removed, the

433 pooled effect remained significant (leave-one-out analysis, range [1.07, 1.15], $p s<.001$ ). See

434 Supplementary Material S7 for more details and figures.

435 Significant heterogeneity was found, $Q(75)=244.06, p<.001$. The $I^{2}$ statistic revealed

436 that $75.35 \%$ of the heterogeneity could not be accounted for by sampling variance. In order to 437 explain this heterogeneity, moderators were examined.

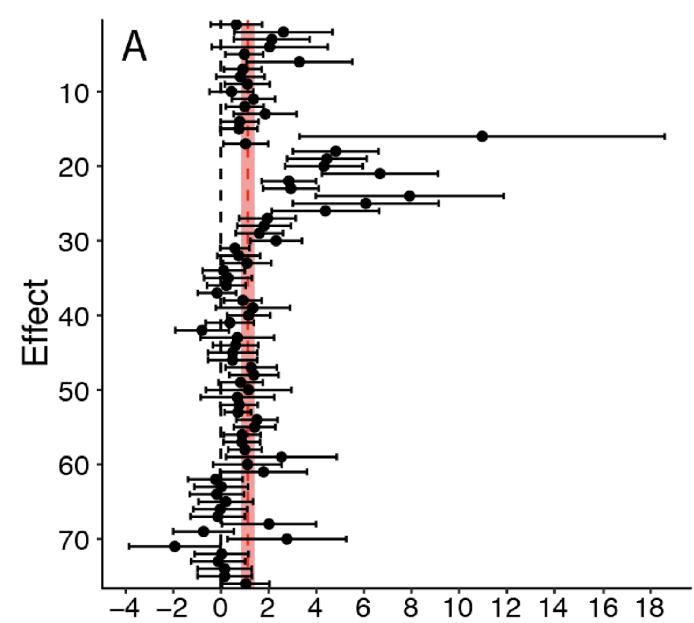

Effect of attention (Cohen's d)

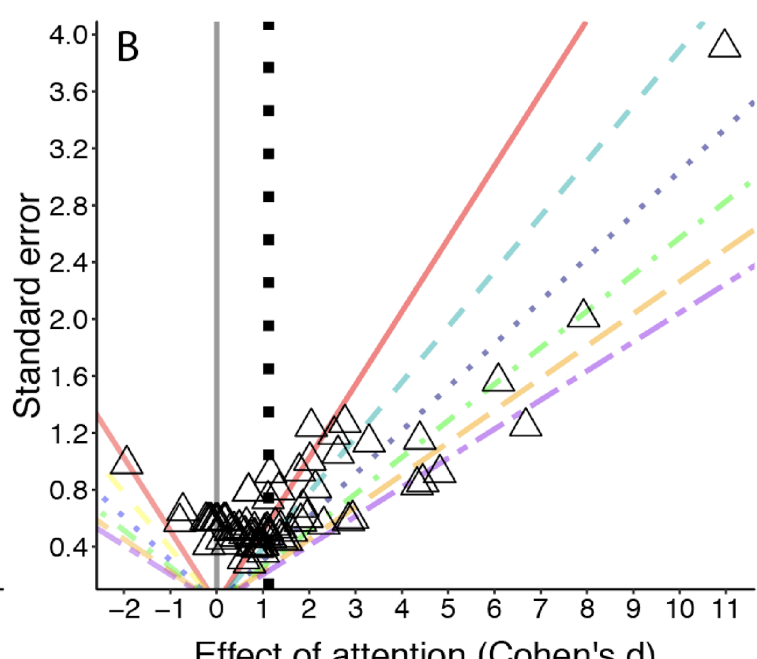

Effect of attention (Cohen's d)

Fig. 2. Summary of the effect of attention on the MAE. a) Forest plot of all 76 effect sizes.

440 Error bars are $95 \%$ confidence intervals. Red dashed line indicates the pooled summary 
441 effect, surrounding shaded area depicts 95\% CI. b) Funnel plot. Dashed line is the pooled

442 effect size, coloured lines represent $p$ values (Purple/Two-dash=.000001, Orange/Long-

$443 \mathrm{dash}=.00001$, Green/Dot-dash=.0001, Blue/Dotted=.001, Turquoise/Dashed=.01,

$444 \mathrm{Red} /$ Solid=.05, Black=1).

445

446

447

3.3. Dependencies: overall analyses

448

A three-tiered model, nesting conditions within independent samples, was a better fit

449 to the data than the two-tiered model $x^{2}(1)=34.69, \mathrm{p}<.001$. This indicates dependence in the

450 data - i.e. there is an effect of study. To characterise the influence of dependency on our

451 global outcomes, the random effects model was applied to 500 resampled data sets, each of

452 which contained one randomly selected effect size from each of our independent samples,

453 yielding a total of 37 effect sizes for each data set. All subsets revealed a significant pooled

454 effect (mean $d=1.01, S D=0.05$ ), indicating a strong attention-MAE relationship regardless of

455 dependency.

456

457

\subsection{Regression models with one moderator}

458

A table summarising all single moderator regression models can be found in

459 Supplementary Material S8. Important moderators (those that were individually significant or

460 contributed to the best multiple regression model) are shown in Fig. 3. Motion type

461 significantly affected the attentional modulation of the $\operatorname{MAE}(Q(1)=17.43, b=-0.99, p<.001)$,

462 accounting for $24.92 \%$ of the total heterogeneity: studies using translating motion stimuli

463 reported significantly larger effects than those using complex motion, (see Fig. 3a). However,

464 attention had a significant effect on motion adaptation within the subset of studies using

465 either translational motion, $(d=1.54, p<.001)$ or complex motion $(d=0.56, p=.002)$. 

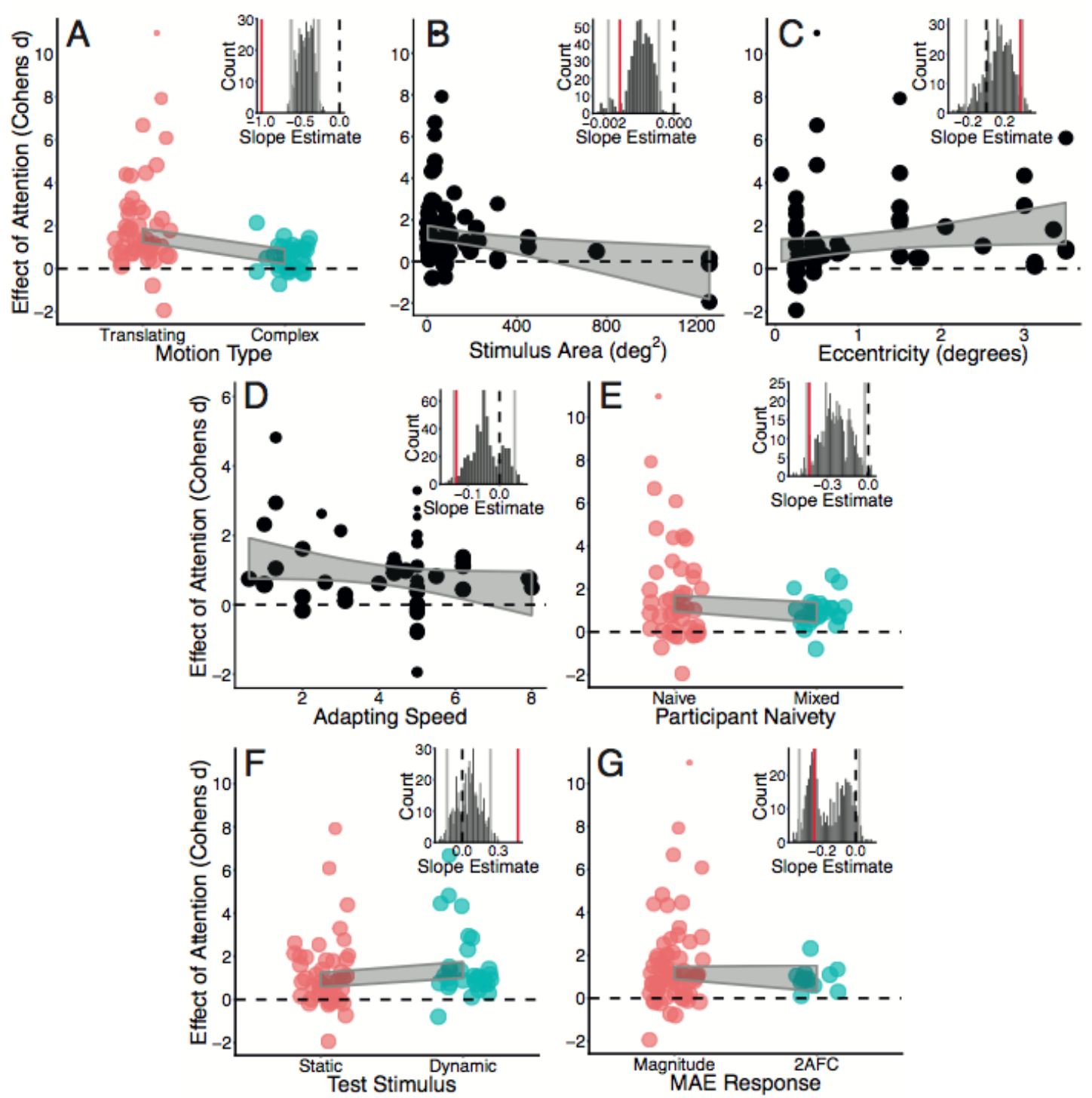

467 Fig. 3. Effect size summary for significant moderators (a-c) and factors that significantly

468 contributed to the final model when considered alongside other moderators (d-g). The effect

469 of attention (a) was larger for translational than complex motion, (b) decreased as stimulus

470 size increased, (c) increased with greater eccentricity, (d) reduced with increasing adaptation

471 speed, (e) was greater for naïve than mixed participant groups, (f) was greater for dynamic

472 vs. static test stimuli, and (g) smaller when using a 2 AFC response. Larger points indicate

473 smaller standard error. The shaded ribbon indicates the 95\% CI from the full dataset. Inset

474 histograms detail the estimated slope parameter for 500 randomly selected datasets with

475 dependency eliminated (see dependency information); red lines indicate estimated slope from 
the full dataset; grey lines indicate the $95 \%$ CI determined by the resampled independent sample estimates; dashed black lines indicate the zero point.

The size (area) of the adaptation stimulus significantly affected attentional modulation of the MAE, $Q(1)=7.40, b=-0.002, p=.007$, accounting for $11.38 \%$ of the heterogeneity in the effect (see Fig. 3b). As stimulus size increased, the magnitude of attentional modulation decreased. In addition, there was a significant effect of the eccentricity of the adapting stimulus, accounting for $8.59 \%$ of the total heterogeneity, $Q(1)=4.05, b=0.37, p=.044$ (see Fig. 3c). The effect of attentional manipulations on the MAE was larger for stimuli that were further from fixation.

When considered alone, the speed of the adaptation stimulus was not significant, $Q(1)=3.36, b=-0.14, p=.067$, accounting for $2.74 \%$ of total heterogeneity. However, motion speed did contribute to the final model (see below); slower stimuli resulted in stronger attentional effects (see Fig. 1d).

Participant characteristics (i.e. naivety) did not significantly moderate the attentionMAE effect in a single moderator model $(Q(1)=1.98, b=-0.43, p=.159)$, but did contribute to the overall model, with larger effects reported for studies using naïve participants. (This was not driven by a relationship between sample size and sample type - see Supplementary Material S9). Significant effects of attention on motion adaptation were found within the subset of studies using naïve participants $(d=1.33, p<.001)$ and within those using experienced, or a mix of naïve and experienced, samples, $(d=0.90, p<.001$; see Fig. 3e). The effect of test stimulus (static vs. dynamic) did not reach significance when considered alone $(Q(1)=3.29, b=0.46, p=.070)$ but did contribute to the best complete model (see below). Using a static test stimulus to assess the MAE resulted in a weaker, though still 
significant, effect of attention $(d=0.91, p<.001)$ than using dynamic tests $(d=1.37, p<.001)$.

501 This accounted for $7.12 \%$ of effect size heterogeneity (Fig. 3f). the MAE. The effect size was similar across studies that measured the MAE duration vs. its strength $(Q(1)=0.49, b=0.19, p=.485)$ with studies employing either method reporting significant effects (MAE duration: $d=1.06, p<.001$; MAE strength: $d=1.24, p<.001$ ). Further, effect size was not significantly modulated by whether a 2AFC design was used vs. a magnitude estimation method $(Q(1)=0.52, b=-0.25, p=.470)$, although the stronger attentional modulation for magnitude designs compared to 2AFC designs contributed to the final model.

509 Both subsets of studies produced significant effects of attention on the MAE (2AFC: $d=.93$, $p=.003$; magnitude estimation: $d=1.17, p<.001$, see Fig. $3 \mathrm{~g}$ ).

513 significant effect of attention was reported within studies that used distractor paradigms (as

514 depicted in Fig. 1b: $d=1.12, p<.001$ ) as well as those that used attentional tracking paradigms (Fig. 1c; $d=1.18, p=.010$ ). The effect size was similar across both (no significant

516 effect of attention paradigm: $Q(1)=0.01, b=0.06, p=.904)$.

As described above, the effect of dependencies was explored via resampling. The

518 distribution of regression coefficients across the 500 resampled sets of independent data can

519 be seen in inset histograms of Fig. 3. Analysing these reduced datasets (with dependencies

520 eliminated) reveals the same set of significant moderators. However, note that the regression

521 coefficient for motion type (translational vs. complex) is larger when estimated from the

522 complete data set than when estimated from reduced datasets. Some of the larger effect sizes

523 for translational motion were produced from samples contributing multiple effects. Thus, we 524 can be confident that studies with translating motion provide substantially larger effect sizes 
525 than those using complex motion, but the estimated magnitude of this difference may be

526 inflated by dependencies across estimates from common subject groups.

527 Selected two-way interaction analyses were conducted, however none of these

528 reached significance (see Supplementary Material S10).

529

530

\subsection{Multiple regression models}

Multiple regression was used to determine the best-fitting model that incorporates

532 multiple factors that contribute significantly to heterogeneity in effect size. A backward

533 elimination strategy was implemented: starting from a model containing all complete effect

534 moderators (those reported for every effect), moderators that did not significantly improve

535 the model were eliminated in a step-wise fashion. We switched to Maximum Likelihood

536 estimation to facilitate model comparison via likelihood ratio tests $(L R T)$. Moderators were

537 assessed in order of significance value, such that the moderator with the largest $p$ value was

538 considered first. Moderators were eliminated when their removal was associated with a non-

539 significant decrease in the goodness of fit of the model, as determined by model comparison

540 via $L R T$. Subsequently, reduced effect moderators (those reported for a subset of effects

541 only) were considered individually and included only if they significantly improved the

542 model. Those with the largest $N$ were assessed first, to maximise the number of cases in the

543 final model.

546 The initial model included all moderators for which values were available for all

547 effects: type of motion (translation vs. complex), MAE measurement (duration vs. strength),

548 MAE response (magnitude vs. 2AFC), test stimulus (static vs. dynamic) and attention

549 paradigm (distractor vs. tracking). Through backward elimination, the optimal complete 
550 effects model included motion type, MAE response and test stimulus as predictors. This

551 model accounted for $42.05 \%$ of the heterogeneity of the effect of attention on motion

552 adaptation.

553

554 3.5.2. Reduced effects

555 The complete effects model was significantly improved by adding four reduced effect

556 moderators. The addition of each one decreases the number of effect sizes $(k)$ included in the

557 model. These reduced effects were participant naivety $(k=69)$, stimulus area $(k=57)$,

558 eccentricity $(k=50)$ and adaptation speed $(k=31)$. The final model accounted for $63.18 \%$ of

559 the heterogeneity of the effect; it is shown in Fig. 4 and summarised in Supplementary

560 Material S11.

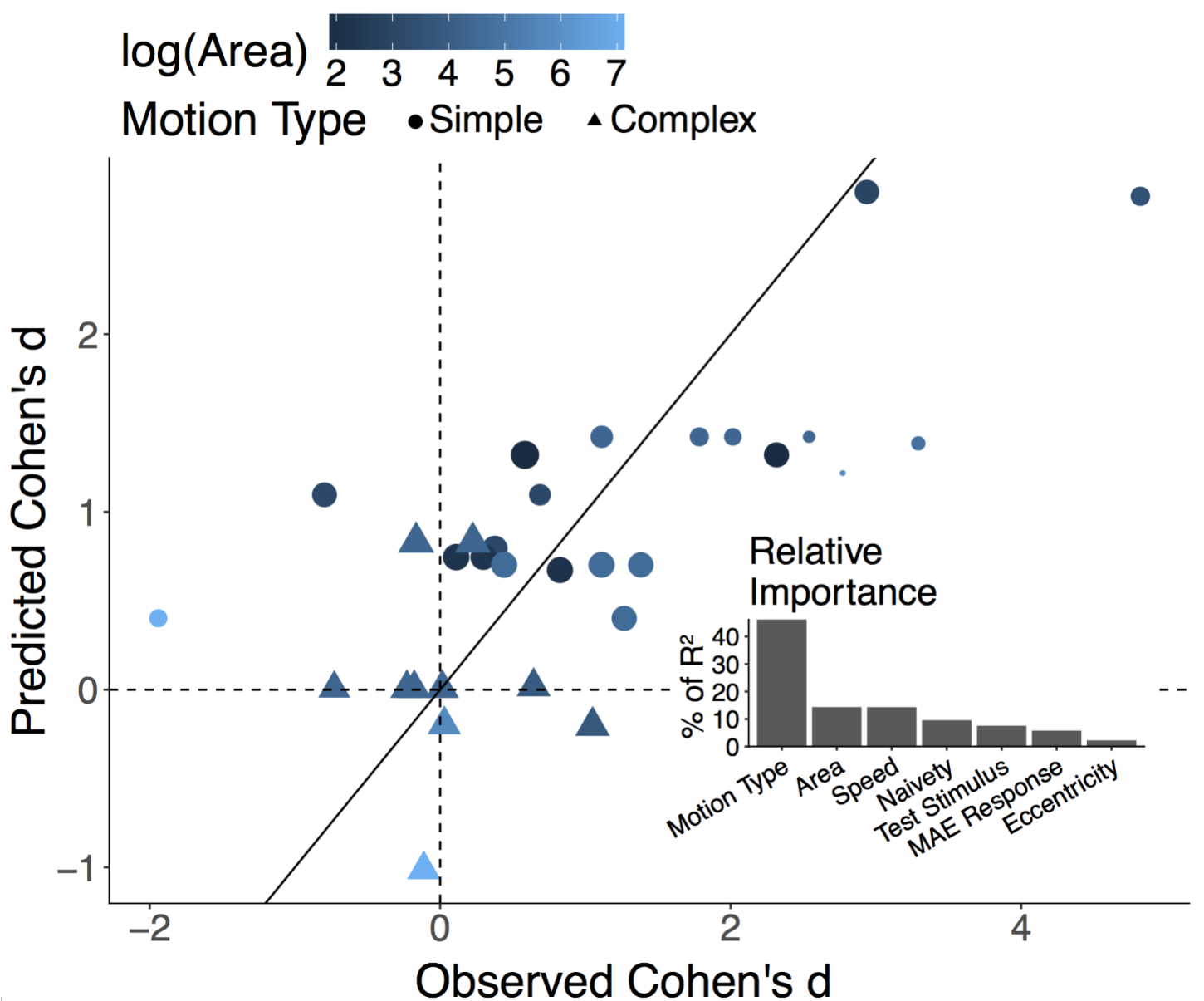

Fig. 4. Predicted Cohen's $d_{A v}$ values as a function of observed Cohen's $d_{A v}$ for each effect size 
563 in final model. Size of the points indicates standard error; larger points have a smaller

564 standard error. Inset: The relative importance of each factor in the final model. This is

565 quantified by the pseudo $\mathrm{R}^{2}$ statistic (see Supplementary Material S5), averaged across all

566 possible orderings of regressor input (Lindeman et al., 1980).

567

568 4. Discussion

569

\subsection{Summary of Findings}

570

Attending to a moving stimulus significantly increases the resultant MAE. This effect

571 of attention is modulated by various characteristics of the adaptation and test stimuli: larger

572 attentional effects were found following adaptation to stimuli that were (i) translating (vs.

573 those with complex motion trajectories) (ii) at a greater eccentricity and (iii) smaller in size.

574 In addition, when considering multiple moderators simultaneously, stronger attentional

575 modulation was reported in studies that employed dynamic, rather than static test stimuli,

576 those using slowly moving adaptation stimuli, and those that used magnitude estimation,

577 rather than $2 \mathrm{AFC}$ judgements to quantify the MAE. Further, greater attentional effects were

578 reported in studies using exclusively naïve participants.

579

580

4.2. Discussion of adaptation and test stimulus characteristics

581

\subsubsection{Type of motion}

582

The effect of attention on motion adaptation was around twice as large for translating motion than for other motion patterns. This behavioural finding suggests that feature-based attention plays a substantial role in increasing motion adaptation. Although translational motion produced substantially stronger attentional effects, adaptation to complex motion was

586 also significantly enhanced by attention. When considered alongside neurophysiology and

587 fMRI evidence, the substantial difference between simple and complex motion suggests that 
surface-based attention may drive some attentional modulation of activity within V1 / MT, but that this effect is small compared to the effects of simple feature-based attention. In addition, we know that neurons in MST can be tuned to large field translating, rotating or

591 expanding / contracting patterns (Smith et al., 2006; Wall et al., 2008). The relatively small 592 effect of attention on adaptation to complex motion therefore also suggests that attentional 593 effects on adaptation within MST are small compared to those in V1/MT. MT/MST but found no significant effects of attention in V1 (Büchel et al., 1998; O'Craven et al., 1997; Watanabe et al., 1998). It is possible that the effects of surface-based attention in V1 are relatively weak and hard to detect. Alternatively, V1 facilitation in a subset of neurons may have been masked by suppressive effects within neurons tuned to unattended motion directions. Facilitatory and suppressive effects of attention (in response to translating stimuli) have recently been revealed in V1 using more sophisticated, voxel-based analyses (Saproo and Serences, 2014), consistent with our findings.

602

603

\subsubsection{Stimulus size}

604

Our analyses revealed a relationship between stimulus size and the effect of attention,

605 with a smaller effect for larger adaptation stimuli, in line with Takeuchi and Kita (1994).

606 They suggested that small vs. large (or whole field) stimuli are processed by separate motion

607 mechanisms, with the former sensitive to object motion, and the latter sensitive to the optic

608 flow generated by self-motion. Our analyses revealed smaller attentional effects for larger

609 adaptation stimuli (which may be processed as optic flow), consistent with the idea that

610 selective attention affects the processing of object, rather than self-motion (Takeuchi \& Kita, 611 1994). 
613 factor of eccentricity - smaller adaptation stimuli might, on average, be positioned further

614 from fixation, allowing better control of spatial attention. Stimulus size was negatively

615 correlated with eccentricity, but this did not reach significance $(r=-0.20, t(51)=-1.45, p=0.15)$.

616 Moreover, both stimulus size and eccentricity contributed significantly to the final model,

617 suggesting that both factors are important in attentional modulation.

618

619

\subsubsection{Eccentricity}

620

Larger effects of attention were found for adaptation stimuli presented at greater

621 distances from fixation. One plausible explanation for this relationship relates to our ability to control spatial attention: when we attend to a central task, the processing of nearby stimuli may also be affected - attention is not perfectly focussed on the central stimulus, but extends

624 to proximal regions.

The positive relationship between eccentricity and the effect of attention on adaptation is in broad agreement with Lavie's (2005) load theory hypothesis - that an increase in load reduces the window of spatial attention.

628

\subsubsection{Test stimuli}

Some researchers have suggested that dynamic and static MAEs correspond to different motion mechanisms (e.g. Verstraten et al., 1996), which may differ in their susceptibility to attention. Our analyses show that attention affects MAEs measured with both dynamic and static test stimuli. Although larger effects were found with dynamic tests, this

634 was a modest difference, that did not reach significance when considered alone and may be an artefact of dependencies within studies (see the resampling analyses in Fig. 3f). 


\subsubsection{Adaptation speed}

Our analyses provide some evidence that the speed of the adaptation stimulus affects attentional modulation of the MAE, with a trend for weaker attentional modulation for faster adaptation stimuli. One possibility is that fast-moving stimuli capture attention, and thus attempts to divert attention are less effective.

\subsection{Factors related to response bias}

\subsubsection{Response type}

We considered whether the effects of attention on the MAE might be driven by, or inflated by, response bias. Asking observers to estimate MAE duration is considered more vulnerable to response bias (Morgan, 2013, 2012, 2011), whereas 2AFC tasks are considered less prone to criterion and / or bias effects. However, reported effect size was not significantly moderated by these factors (when considered in single predictor models). In fact, measures of MAE magnitude produced slightly larger estimates of the attentional effect than studies that asked observers to report MAE duration. Although the choice of 2AFC vs. magnitude estimation was not significant when considered alone, it did contribute to the final model, providing some evidence that $2 \mathrm{AFC}$ paradigms produce slightly more conservative (but still significant) estimates of the attentional effect.

\subsubsection{Participant characteristics}

If response bias does inflate estimates of the effect of attention, one would expect this to be an issue predominantly amongst non-naïve observers, who understand the attention hypothesis: for response bias to modulate effect size, observers should not only bias their responses in accordance with motion adaptation, but to systematically vary this bias as a function of attention condition. On the contrary, participant naivety did not reach significance 
as a single moderator, and experiments using naïve subjects reported larger effects, with this effect contributing to the final model.

664

665

\subsection{Nonsignificant moderators}

A strong and significant effect of attention was found within both distractor and attentional tracking paradigms; both provide an effective manipulation of attention, and modulate the MAE to a similar extent. This contrasts with the suggestion that the two paradigms probe distinct attentional mechanisms, and that only attentional tracking paradigms modulate motion adaptation (Morgan, 2012, 2011). An alternative, supported by our analysis, is that attentional tracking paradigms have produced more consistent effects of attention because they tend to use translating motion stimuli (rather than expanding, or rotating motion). not significantly moderate attentional modulation of the MAE. Previously we examined whether attention affects the rate at which the MAE builds up, or the asymptotic MAE. We found that attention affects the MAE asymptote, rather than the timecourse of adaptation, and this finding is mirrored in our current analysis: comparable attentional modulation was discovered across the wide range of adaptation durations included in the surveyed literature. Prior work has demonstrated that MAEs increase with adaptation duration (e.g. Bartlett et al., 2018). As discussed above, this duration-related increase in MAEs is not associated with increased attentional modulation. However, it is worth considering whether, more broadly, larger MAEs are associated with increased attentional modulation. Could it be

684 that study designs that produce large MAEs (in terms of effect size) are more sensitive, and 685 therefore also report greater effects of attentional modulation? Moreover, could MAE strength be a mediating variable that 'explains' the effects of some of our identified 
687

688

689

690

691

692

693

694

695

696

697

698

699

700

701

702

703

704

705

706

707

708

709

710

711

moderators? To investigate this, we quantified baseline MAE strength (in the passive, or lowload condition) using Cohen's D. (Equation 1 reduces to the mean difference between a null result (i.e. no MAE) and the low-load / passive condition, divided by the low-load standard deviation). A small number of effects (12) were excluded from this analysis because an estimate of baseline MAE strength was not available. Within the remaining effects $(k=64)$, MAE strength was not substantially or significantly related to the effect of attention $(Q(1)=0.20, b=-0.02, p=.655)$. Further analyses confirmed that MAE strength did not significantly contribute to the final model, $L R T=0.06, p=.800(k=57)$. Thus, significant predictors of the modulatory effect of attention on motion adaptation (such as motion type, or stimulus size) cannot be 'explained away' via effects on baseline MAE strength.

\subsection{Reconciling the literature}

Attentional modulation of the MAE is a fairly robust effect: significant effects were found at each level of all categorical moderators. However, the strength of the attention effect is substantially moderated by a number of factors, with motion type (translating vs. complex) being the most important. Our multi-moderator model accounted for $63.18 \%$ of variation in effect size across studies.

Other, un-modelled factors will also contribute to apparent inconsistencies in the literature. For example, within the distractor paradigm, studies vary in how they manipulate attention; some 'high load' or 'diverted' attention conditions may be less effective than others in drawing attention away from the motion stimulus. However, this variability is hard to model, given the variety of tasks, and - in some studies - a lack of information about task difficulty / observer accuracy.

\subsection{Relationship to neuroimaging}


713 provided evidence that attention modulates motion-related activity at multiple cortical

714 regions, including V1, MT and MST. Although early work focussed on spatial attention,

715 many studies have revealed effects of feature-based attention, and surface-based attention.

716 Some have suggested that a unified attention system exists that treats stimulus location (and 717 possibly object identity) as stimulus 'features', alongside motion direction (see Maunsell and

718 Treue, 2006), although recent work suggests that spatial and feature-based attention may, in 719 part at least, rely on different underlying neural mechanisms (Xue et al., 2017). Our meta720 analysis suggests that simple, feature-based attention has much stronger effects on motion 721 adaptation than spatial attention, or higher-level 'surface-based' attention: the largest effects 722 of attention are seen for coherently translating stimuli, that maximise the effects of feature723 based attention. This suggests a larger role for V1 and MT in attentional modulation of 724 motion processing.

725 Attentional modulation decreased for larger stimuli at smaller eccentricities, conditions that may increase the extent to which spatial attention 'spills over' from a central task, to a peripheral motion stimulus. This makes sense if spatial attention modulates neuronal activity according to the extent of overlap between a neuron's receptive field and 729 the spatial locus of attention (Maunsell and Treue, 2006). Large-field stimuli will be 730 particularly effective in driving activation in MSTd, where receptive fields are large and more will overlap with a central attended region of visual space.

733 stimuli. Previous authors have shown greater interocular transfer of the dynamic MAE

734 (Nishida et al., 1994) and suggested that static MAEs predominantly reflect adaptation in V1, 735 whereas dynamic MAEs also involve MT (Mather et al., 2008). Our findings are broadly 
consistent with this suggestion - the larger effect of attention on the dynamic MAE may reflect attentional modulation across V1 and MT/MST.

738

\section{Conclusions}

Our meta-analysis supports a number of conclusions. First, there is overwhelming

741 evidence that motion adaptation is affected by attention. By analysing the effects of different

742 paradigms, participant naivety and looking for evidence of the 'file drawer' effect, we can be

743 confident that reported effects are not driven by response bias or publication bias. It seems

744 that Wohlgemuth (1911) was wrong after all. More importantly, we identified several factors

745 that modulate the effects of attention on motion adaptation, allowing us to explain some

746 apparent inconsistencies in the literature. Our analyses suggest that the largest effects of

747 attention on motion adaptation will be seen for studies that use translating motion stimuli,

748 within either attentional tracking paradigms, or 'distraction' paradigms, particularly when the

749 adaptation stimuli are some distance from fixation. These design choices are likely to exploit

750 (direction-specific) feature-based attention, largely reflected in V1 and MT modulation that

751 includes both facilitation and suppressive effects. However, some additional attentional

752 modulation is likely to be driven by surface-based attention and (for 'distraction' paradigms)

753 spatial attention.

754

755

756

757

758

759

760 
References

762

763 References marked with an asterisk indicate studies included in the meta-analysis.

764

765 Addams, R., 1834. An account of a peculiar optical phenomenon seen after having looked at 766 a moving body. London Edinburgh Philos. Mag. J. Sci. 5, 373-374.

767 https://doi.org/10.1080/14786443408648481

768

*Aghdaee, S.M., Zandvakili, A., 2005. Adaptation to spiral motion: Global but not local 769

770 motion detectors are modulated by attention. Vision Res. 45, 1099-1105.

771 https://doi.org/10.1016/j.visres.2004.11.012

*Alais, D., Blake, R., 1999. Neural strength of visual attention gauged by motion adaptation.

772 Nat. Neurosci. 2, 1015-1018. https://doi.org/10.1038/14814

773

Arizona Software Inc., 2010. GraphClick 3.0.2.

774 Bartlett, L., Graf, E.W., Adams, W.J., 2018. The effects of attention and adaptation duration 775 on the motion aftereffect. J. Exp. Psychol. Hum. Percept. Perform. Advance online publication. https://doi.org/10.1037/xhp0000572

777 Beauchamp, M.S., Cox, R.W., DeYoe, E.A., 1997. Graded effects of spatial and featural 778 779

Becker, B.J., 2005. Failsafe N or file-drawer number, in: Rothstein, H.R., Sutton, A.J., 781 Borenstein, M. (Eds.), Publication Bias in Meta-Analysis: Prevention, assessment and adjustments. John Wiley \& Sons., Chichester, pp. 111-125

783 Begg, C.B., Berlin, J.A., 1988. Publication bias: A problem in interpreting medical data. Journal of the Royal Statistical Society. Series A (Statistics in Society) 3, 419-463. https://doi.org/10.2307/2982993 
786

787

788

789

790

791

792

793

794

795

796

797

798

799

800

801

802

803

804

805

806

807

808

809

810

Begg, C.B., Mazumdar, M. 1994. Operating characteristics of a rank correlation test for publication bias. Biometrics 50, 1088-1101. https://doi.org/10.2307/2533446

*Berman, R.A., Colby, C.L., 2002. Auditory and visual attention modulate motion processing in area MT+. Cogn. Brain Res. 14, 64-74. https://doi.org/10.1016/S09266410(02)00061-7

Blake, R., Fox, R., 1974. Adaptation to invisible gratings and the site of binocular rivalry suppression. Nature 249, 488-490. https://doi.org/10.1038/249488a0

Blake, R., Hiris, E., 1993. Another means for measuring the motion aftereffect. Vision Res. 33, 1589-1592. https://doi.org/10.1016/0042-6989(93)90152-M

Blake, R., Tadin, D., Sobel, K. V., Raissian, T.A., Chong, S.C., 2006. Strength of early visual adaptation depends on visual awareness. Proc. Natl. Acad. Sci. U. S. A. 103, 47834788. https://doi.org/10.1073/pnas.0509634103

*Blaser, E., Shepard, T., 2009. Maximal motion aftereffects in spite of diverted awareness. Vision Res. 49, 1174-1181. https://doi.org/10.1016/j.visres.2008.09.012

*Büchel, C., Josephs, O., Rees, G., Turner, R., Frith, C.D., Friston, K.J., 1998. The functional anatomy of attention to visual motion. A functional MRI study. Brain 121, 1281-1294. https://doi.org/10.1093/brain/121.7.1281

Burr, D., Thompson, P., 2011. Motion psychophysics: 1985-2010. Vision Res. 51, 14311456. https://doi.org/10.1016/j.visres.2011.02.008

Carrasco, M., 2011. Visual attention: The past 25 years. Vision Res. 51, 1484-1525. https://doi.org/10.1016/j.visres.2011.04.012

*Chaudhuri, A., 1990. Modulation of the motion aftereffect by selective attention. Nature 344, 60-62. https://doi.org/10.1038/344060a0

Cheung, M.W.-L., 2014. Modeling dependent effect sizes with three-level meta-analyses: A structural equation modeling approach. Psychol. Methods 19, 211-229. 
812 Cohen, J., 1977. Statistical power analysis for the behavioral sciences. Academic Press, Inc., 813 New York. https://doi.org/10.1016/B978-0-12-179060-8.50001-3

814 *Culham, J.C., Verstraten, F.A.J., Ashida, H., Cavanagh, P., 2000. Independent aftereffects 815 of attention and motion. Neuron 28, 607-615. https://doi.org/10.1016/S0896-

$816 \quad 6273(00) 00137-9$

817 Cumming, G., 2012. Understanding the new statistics. Routledge, New York.

818 *Dobkins, K.R., Rezec, A.A., Krekelberg, B., 2007. Effects of spatial attention and salience 819 cues on chromatic and achromatic motion processing. Vision Res. 47, 1893-1906.

820 https://doi.org/10.1016/j.visres.2006.12.021

821 Driver, J., McLeod, P., Dienes, Z., 1992. Motion coherence and conjunction search:

822 Implications for guided search theory. Percept. Psychophys. 51, 79-85.

823 https://doi.org/10.3758/BF03205076

824 Duval, S., Tweedie, R., 2000a. A nonparametric “Trim and Fill" method of accounting for 825 publication bias in meta-analysis. J. Am. Stat. Assoc. 95, 89-98.

826 https://doi.org/10.1080/01621459.2000.10473905

827 Duval, S., Tweedie, R., 2000b. Trim and fill: A simple funnel-plot-based method of testing 828 and adjusting for publication bias in meta-analysis. Biometrics 56, 455-463.

829 https://doi.org/10.1111/j.0006-341x.2000.00455.x

830 Egger, M., Smith, G.D, Schneider, M., Minder, C. 1997. Bias in meta-analysis detected by a 831 simple, graphical test. British Medical Journal 315, 629-634.

832 https://doi.org/10.1136/bmj.315.7109.629

833 Frisby, J.P., 1979. Seeing: Illusion, Brain and Mind. Oxford University Press.

$834 *$ Georgiades, M.S., Harris, J.P., 2002a. Effects of attentional modulation of a stationary 835 surround in adaptation to motion. Perception 31, 393-408. https://doi.org/10.1068/p3199 
*Georgiades, M.S., Harris, J.P., 2002b. Evidence for spatio-temporal selectivity in attentional modulation of the motion aftereffect. Spat. Vis. 16, 21-31. https://doi.org/10.1163/15685680260433887

*Georgiades, M.S., Harris, J.P., 2000a. Attentional diversion during adaptation affects the velocity as well as the duration of motion after-effects. Proc. R. Soc. London B Biol. Sci. 267, 2559-2565. https://doi.org/10.1098/rspb.2000.1321

*Georgiades, M.S., Harris, J.P., 2000b. The spatial spread of attentional modulation of the motion aftereffect. Perception 29, 1185-1201. https://doi.org/10.1068/p3023

844 Gibson, J.J., 1958. Visually controlled locomotion and visual orientation in animals. Br. J. 845 Psychol. 49, 182-194. https://doi.org/10.1111/j.2044-8295.1958.tb00656.x

846 Graziano, M.S.A., Andersen, R.A., Snowden, R.J., 1994. Tuning of MST neurons to spiral 847 motions. J. Neurosci. 14, 54-67.

848 Greenhouse, J.B., Iyengar, S., 1994. Sensitivity analysis and diagnostics, in: Cooper, H., 849 Hedges, L. V. (Eds.), The Handbook of Research Synthesis. Russell Sage Publications, $850 \quad$ New York, pp. 417-434.

851 He, S., Cavanagh, P., Intriligator, J., 1996. Attentional resolution and the locus of visual 852 awareness. Nature 383, 334-337. https://doi.org/10.1038/383334a0

853 Hershenson, M., 1993. Linear and rotation motion aftereffects as a function of inspection 854

*Houghton, R.J., Macken, W.J., Jones, D.M., 2003. Attentional modulation of the visual motion aftereffect has a central cognitive locus: Evidence of interference by the postcategorical on the precategorical. J. Exp. Psychol. Hum. Percept. Perform. 29, 731740. https://doi.org/10.1037/0096-1523.29.4.731

859 *Jung, Y., Chong, S.C., 2014. Effects of attention on visible and invisible adapters.

860 Perception 43, 549-568. https://doi.org/10.1068/p7660 
861 Kamitani, Y., Tong, F., 2006. Decoding seen and attended motion directions from activity in

862 the human visual cortex. Curr. Biol. 16, 1096-1102.

863 https://doi.org/10.1016/j.cub.2006.04.003

864 *Kaunitz, L., Fracasso, A., Melcher, D., 2011. Unseen complex motion is modulated by

865 attention and generates a visible aftereffect. J. Vis. 11, 1-9.

$866 \quad$ https://doi.org/10.1167/11.13.10

867 Kohler, A., Haddad, L., Singer, W., Muckli, L., 2008. Deciding what to see: The role of

868 intention and attention in the perception of apparent motion. Vision Res. 48, 1096-1106.

869 https://doi.org/10.1016/j.visres.2007.11.020

870 Kohn, A., Movshon, J.A., 2004. Adaptation changes the direction tuning of macaque MT

871 neurons. Nat. Neurosci. 7, 764-772. https://doi.org/10.1038/nn1267

872 Kohn, A., Movshon, J.A., 2003. Neuronal adaptation to visual motion in area MT of the

873 macaque. Neuron 39, 681-691. https://doi.org/10.1016/S0896-6273(03)00438-0

874 Lakens, D., 2013. Calculating and reporting effect sizes to facilitate cumulative science: A

875 practical primer for t-tests and ANOVAs. Front. Psychol. 4, 1-12.

876 https://doi.org/10.3389/fpsyg.2013.00863

877 Lankheet, M.J.M., Verstraten, F.A.J., 1995. Attentional modulation of adaptation to two-

878 component transparent motion. Vision Res. 35, 1401-1412.

879 https://doi.org/10.1016/0042-6989(95)98720-T

880 Lavie, N., 2005. Distracted and confused?: Selective attention under load. Trends Cogn. Sci.

8819 9, 75-82. https://doi.org/10.1016/j.tics.2004.12.004

882 Lindeman, R.H., Merenda, P.F., Gold, R.Z., 1980. Introduction to bivariate and multivariate 883 analysis. Scott, Foresman, Glenview, IL.

884 López-López, J.A., Marín-Martínez, F., Sánchez-Meca, J., Van den Noortgate, W.,

885 Viechtbauer, W., 2014. Estimation of the predictive power of the model in mixed-effects 
888 Lu, Z.L., Sperling, G., 2001. Three-systems theory of human visual motion perception:

889 review and update. J. Opt. Soc. Am. A 18, 2331-2370.

$890 \quad$ https://doi.org/10.1364/JOSAA.18.002331

891 Lu, Z.L., Sperling, G., 1995. The functional architecture of human visual motion perception.

892 Vision Res. 35, 2697-2722. https://doi.org/10.1016/0042-6989(95)00025-U

893 Mather, G., Pavan, A., Campana, G., Casco, C., 2008. The motion aftereffect reloaded.

894 Trends Cogn. Sci. 12, 481-487. https://doi.org/10.1016/j.tics.2008.09.002

895 Maunsell, J.H.R., Treue, S., 2006. Feature-based attention in visual cortex. Trends Neurosci.

$89629,317-322$. https://doi.org/10.1016/j.tins.2006.04.001

897 Moher, D., Liberati, A., Tetzlaff, J., Altman, D.G., The PRISMA Group, 2009. Preferred

898 reporting items for systematic reviews and meta-analyses: The PRISMA statement.

899 PLoS Med. 6, 1-6. https://doi.org/10.1371/journal.pmed.1000097

900 *Morgan, M.J., 2013. Sustained attention is not necessary for velocity adaptation. J. Vis. 13,

$901 \quad 1-11$. https://doi.org/10.1167/13.8.26

902 *Morgan, M.J., 2012. Motion adaptation does not depend on attention to the adaptor. Vision

903 Res. 55, 47-51. https://doi.org/10.1016/j.visres.2011.12.009

904 Morgan, M.J., 2011. Wohlgemuth was right: Distracting attention from the adapting stimulus

905 does not decrease the motion after-effect. Vision Res. 51, 2169-2175.

906 https://doi.org/10.1016/j.visres.2011.07.018

907 Morris, S.B., DeShon, R.P., 2002. Combining effect size estimates in meta-analysis with

908 repeated measures and independent-group designs. Psychol. Methods 7, 105-125.

909 https://doi.org/10.1037//1082-989X.7.1.105

910 *Mukai, I., Watanabe, T., 2001. Differential effect of attention to translation and expansion 
913 Nakayama, K., 1985. Biological image motion processing: A review. Vision Res. 25, 625914 660. https://doi.org/10.1016/0042-6989(85)90171-3

915 Nakayama, K., Silverman, G.H., 1986. Serial and parallel processing of visual feature 916 conjunctions. Nature 320, 264-265. https://doi.org/10.1038/320264a0

917 *Nishida, S., Ashida, H., 2000. A hierarchical structure of motion system revealed by 918 interocular transfer of flicker motion aftereffects. Vision Res. 40, 265-278.

919 https://doi.org/10.1016/S0042-6989(99)00176-5

920 Nishida, S., Ashida, H., Sato, T., 1994. Complete interocular transfer of motion aftereffect 921 with flickering test. Vision Res. 34, 2707-2716. https://doi.org/10.1016/00426989(94)90227-5

923 Nishida, S., Sato, T., 1995. Motion aftereffect with flickering test patterns reveals higher 924 stages of motion processing. Vision Res. 35, 477-490. https://doi.org/10.1016/0042$925 \quad 6989(94) 00144-B$

926 O’Craven, K.M., Rosen, B.R., Kwong, K.K., Treisman, A., Savoy., R.L., 1997. Voluntary 927 attention modulates fMRI activity in human MT-MST. Neuron 18, 591-598.

928 https://doi.org/10.1016/S0896-6273(00)80300-1

929 Orwin, R.G. 1983. A fail-safe N for effect size in meta-analysis. J. Educ. Behav. Stat. 8, 157930 159. https://doi.org/10.3102/10769986008002157

931 *Patterson, R., Fournier, L.R., Wiediger, M., Vavrek, G., Becker-Dippman, C., Bickler, I., 932 2005. Selective attention and cyclopean motion processing. Vision Res. 45, 2601-2607. 933 https://doi.org/10.1016/j.visres.2005.03.003

934 Pavan, A., Greenlee, M.W., 2015. Effects of crowding and attention on high-levels of motion 935 processing and motion adaptation. PLoS One 10, 1-27. 
*Rees, G., Frith, C., Lavie, N., 2001. Processing of irrelevant visual motion during

938 performance of an auditory attention task. Neuropsychologia 39, 937-949.

939 https://doi.org/10.1016/S0028-3932(01)00016-1

940 *Rees, G., Frith, C.D., Lavie, N., 1997. Modulating irrelevant motion perception by varying

941 attentional load in an unrelated task. Science 278, 1616-1619.

942 https://doi.org/10.1126/science.278.5343.1616

943 *Rezec, A., Krekelberg, B., Dobkins, K.R., 2004. Attention enhances adaptability: Evidence

944 from motion adaptation experiments. Vision Res. 44, 3035-3044.

$945 \quad$ https://doi.org/10.1016/j.visres.2004.07.020

946 *Rose, D., Bradshaw, M.F., Hibbard, P.B., 2003. Attention affects the stereoscopic depth

947 aftereffect. Perception 32, 635-640. https://doi.org/10.1068/p3324

948 Rosenthal, R., 1991. Meta-analytic procedures for social research (revised edition), SAGE

949 Publications. Newbury Park, CA.

950 Saito, H., Yukie, M., Tanaka, K., Hikosaka, K., Fukada, Y., Iwai, E., 1986. Integration of

951 direction signals of image motion in the superior temporal sulcus of the macaque

952 monkey. J. Neurosci. 6, 145-157.

953 Saproo, S., Serences, J.T., 2014. Attention improves transfer of motion information between

954 V1 and MT. J. Neurosci. 34, 3586-3596. https://doi.org/10.1523/JNEUROSCI.3484-

$955 \quad 13.2014$

956 *Shioiri, S., Matsumiya, K., 2009. Motion mechanisms with different spatiotemporal

957 characteristics identified by an MAE technique with superimposed gratings. J. Vis. 9, 1-

$958 \quad$ 15. https://doi.org/10.1167/9.5.30

$959 *$ Shulman, G.L., 1993. Attentional effects on adaptation of rotary motion in the plane.

$960 \quad$ Perception 22, 947-961. https://doi.org/10.1068/p220947 
961

962

963

964 Silver, M.A., Ress, D., Heeger, D.J., 2007. Neural correlates of sustained spatial attention in 965 human early visual cortex. J. Neurophysiol. 97, 229-237.

966

967

968

969

*Shulman, G.L., 1991. Attentional modulation of mechanisms that analyze rotation in depth. J. Exp. Psychol. Hum. Percept. Perform. 17, 726-737. https://doi.org/10.1037/00961523.17.3.726

https://doi.org/10.1152/jn.00677.2006

Smith, A.T., Singh, K.D., Williams, A.L., Greenlee, M.W., 2001. Estimating receptive field size from fMRI data in human striate and extrastriate visual cortex. Cereb. Cortex 11, 1182-1190. https://doi.org/10.1093/cercor/11.12.1182

Smith, A.T., Wall, M.B., Williams, A.L., Singh, K.D., 2006. Sensitivity to optic flow in human cortical areas MT and MST. Eur. J. Neurosci. 23, 561-569.

https://doi.org/10.1111/j.1460-9568.2005.04526.x

Somers, D.C., Dale, A.M., Seiffert, A.E., Tootell, R.B.H., 1999. Functional MRI reveals spatially specific attentional modulation in human primary visual cortex. Proc. Natl. Acad. Sci. USA 96, 1663-1668. https://doi.org/10.1073/pnas.96.4.1663

*Takeuchi, T., Kita, S., 1994. Attentional modulation in motion aftereffect. Jpn. Psychol. Res. 36, 94-107.

Tanaka, K., Saito, H., 1989. Analysis of motion of the visual field by direction,

expansion/contraction, and rotation cells clustered in the dorsal part of the medial superior temporal area of the macaque monkey. J. Neurophysiol. 62, 626-641.

https://doi.org/10.1152/jn.1989.62.3.626

*Taya, S., Adams, W.J., Graf, E.W., Lavie, N., 2009. The fate of task-irrelevant visual motion: Perceptual load versus feature-based attention. J. Vis. 9, 1-10.

https://doi.org/10.1167/9.12.12

Thompson, P., 1998. Tuning of the motion aftereffect, in: Mather, G., Verstraten, F., Anstis, 
Treue, S., Martínez-Trujillo, J.C., 1999. Feature-based attention influences motion processing gain in macaque visual cortex. Nature 399, 575-579. https://doi.org/10.1038/21176

Treue, S., Maunsell, J.H.R., 1996. Attentional modulation of visual motion processing in cortical areas MT and MST. Nature 382, 539-541. https://doi.org/10.1038/382539a0

Verstraten, F.A.J., Fredericksen, R.E., Van Wezel, R.J.A., Lankheet, M.J.M., Van De Grind, 6989(95)00111-5

Viechtbauer, W., 2010. Conducting meta-analyses in R with the metafor package. J. Stat. Softw. 36, 1-48.

997

Viechtbauer, W., 2005. Bias and efficiency of meta-analytic variance estimators in the random-effects model. J. Educ. Behav. Stat. 30, 261-293.

Wall, M.B., Lingnau, A., Ashida, H., Smith, A.T., 2008. Selective visual responses to expansion and rotation in the human MT complex revealed by functional magnetic resonance imaging adaptation. Eur. J. Neurosci. 27, 2747-2757.

1003 https://doi.org/10.1111/j.1460-9568.2008.06249.x

1004 Wannig, A., Rodríguez, V., Freiwald, W.A., 2007. Attention to surfaces modulates motion 1005 processing in extrastriate area MT. Neuron 54, 639-651. https://doi.org/10.1016/j.neuron.2007.05.001

1007 Watanabe, T., Harner, A.M., Miyauchi, S., Sasaki, Y., Nielsen, M., Palomo, D., Mukai, I., 1008 1998. Task-dependent influences of attention on the activation of human primary visual cortex. Proc. Natl. Acad. Sci. U. S. A. 95, 11489-11492.

$$
\text { https://doi.org/10.1073/pnas.95.19.11489 }
$$


1011 Wertheimer, M., 1961. Experimental studies on the seeing of motion, in: T.Shipley (Ed.), 1012 Classics in Psychology. Philosophical Library, New York, pp. 1032-1089.

1013 Wohlgemuth, A., 1911. On the after-effect of seen motion. Br. J. Psychol. 1, 1-117.

1014 Xue, C., Kaping, D., Ray, S.B., Krishna, B.S., Treue, S., 2017. Spatial attention reduces 1015 burstiness in Macaque visual cortical area MST. Cereb. Cortex 27, 83-91.

1016 https://doi.org/10.1093/cercor/bhw326

1017 